\title{
LASER POWER MODULATION DIRECT OVERWRITE ON A CO/Pt MULTILAYER
}

\author{
A.KIKITSU and K.ICHIHARA \\ Toshiba Corp., R\&D Center \\ 1 Komukai Toshiba-cho, Saiwai-ku, Kawasaki 210, Japan
}

Abstract- Laser power modulation dircet overwrite (OW) on a $\mathrm{Co} / \mathrm{Pt}$ multilayer was successful by the magneto-statically coupled $\mathrm{OW}$ method. In order to use the $\mathrm{Co} / \mathrm{Pt}$ multilayer as the storage layer in this method, the magnetization reversal temperature was adjusted by changing the thickness of the Co layer and a small switching field was accomplished by making the total thickness thinner. An OW CNR up to $32 \mathrm{~dB}$ with the conventional rare earth-transition metal biasing layer was obtained for the $\mathrm{Co} / \mathrm{Pt}$ multilayers stacked 2 or 4 times.

KEYWORDS: OVERWRITE,POWER MODULATION,LEAKAGE FIELD,Co/Pt MULTILAYER

\section{INTRODUCTION}

Direct overwrite (OW) is the most effective approach for improving the data transfer rate during writing in a magneto-optical (MO) recording system. A new laser power modulation OW method, in which overwriting was carried out by modulating the leakage field generated in the medium, was proposed in a previous work[1]. In this method, the main functional layers, that is, the storage layer (SL) and the biasing layer (BL), were coupled magneto-statically and an initializing process was not necessary before $O W$. Therefore, an MO recording material which has a low coercivity $(\mathrm{Hc})$ less than $1 \mathrm{kOe}$ can be used for an SL. This is one of the advantages of the magneto-statically coupled OW method.

A thin film $\mathrm{Co} / \mathrm{Pt}$ multilayer has been studied as a new MO recording material[2,3]. $\mathrm{Co} / \mathrm{Pt}$ multilayers are thought to be a good candidate for a high density MO recording medium because they have a larger Kerr rotation angle than conventional rear-earth transition metal alloy films at short wavelength. If OW technology is applied to this $\mathrm{Co} / \mathrm{Pt}$ material, a high speed and high density MO recording system will be realized. For practical use, Co/Pt has a disadvantage of low $\mathrm{Hc}$, but this problem is not so serious for the magneto-statically coupled OW method in principle.

In this paper, an experimental result of laser power modulation $\mathrm{OW}$ on a $\mathrm{Co} / \mathrm{Pt}$ multilayer is shown. Several modifications were required on the magnetic characteristics of $\mathrm{Co} / \mathrm{Pt}$ when it was applied to the storage layer in the magneto-statically coupled OW medium. The result of these modifications are also presented.

\section{OW PRINCIPLE AND REQUIREMENTS FOR THE SL}

The cross-sectional view of the $\mathrm{OW}$ medium is shown in Fig.1. It consists of four functional layers; SL, thermal control layer (TCL), BL, and initializing layer (IL). A conceptual view of OW process is shown in Fig.2. In this figure, 2-dimensional profiles of the leakage field $(\mathrm{HI})$ and the isotherm of the magnetization reversal temperature in SL (assumed as the Curie temperature of SL ; Tcs) are shown. (a) in the write mode (high power irradiation) and (b) in the crase mode (low power irradiation) are the time when the temperature of SL reaches its maximum value. The medium is cooled from state (a) or (b) to state (d), and $\mathrm{Hl}$ at the Tcs isotherm increases in time by the thermal diffusion between SL and $\mathrm{BL} . \mathrm{Hl}$ in the write mode becomes larger than $\mathrm{HI}$ in the erase mode because the difference in temperature between BL and SL becomes small. Therefore, the direction of the magnetic field at the Tcs isotherm can be modulated between plus and minus by adding an appropriate external DC field (Hex), and a

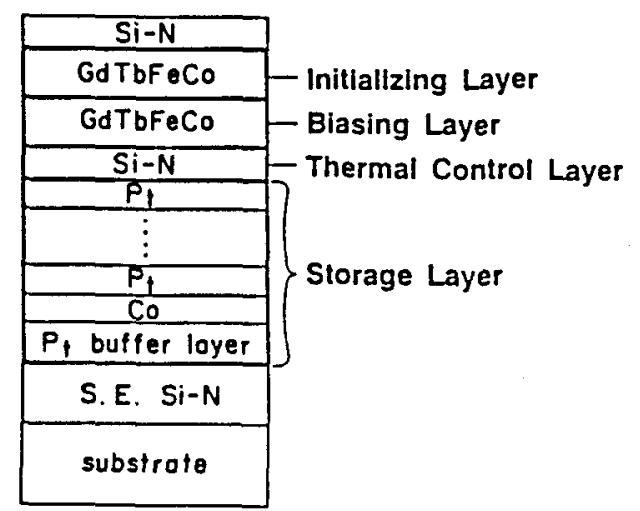

Fig.1 Schematic cross-sectional view of overwrite medium 
reversed magnetic domain, which is indicated as the hatched area in Fig.2, is created or erased by the laser power level.

The following qualifications are required for SL in order to carry out overwrite successfully.

(1) The magnetization reversal tempcrature $(\mathrm{Tr})$ is required to be adjusted in order to achicve a large leakage ficld modulation. It is preferable for $\mathrm{Tr}$ to be nearly equal to the Curie temperature of the $\mathrm{BL}(\mathrm{TcB})$, typically $200^{\circ} \mathrm{C}$ $[1,4]$.

(2) Writing and erasing are required to be completed with as small a change in Hex as possible, because the degree of leakage field modulation was evaluated to be about $150 \mathrm{Oe}$ for the OW medium used in a previous work [4]. This Hex change is defined as the switching field $\Delta \mathrm{Hsw}$ in this paper.

(3) The lower limit of $\mathrm{Hc}$ is required to be larger than about $200 \mathrm{Oe}$, because the external field necessary for $O W$ is considered to be within $\pm 200 \mathrm{Oe}[4]$. In this paper, the nucleation coercive force, $\mathrm{Hcn}$, was investigated for this requirement, because it represents the coercive force to prevent the collapse of the reversed magnetic domain by the external field.

Write mode Erose mode

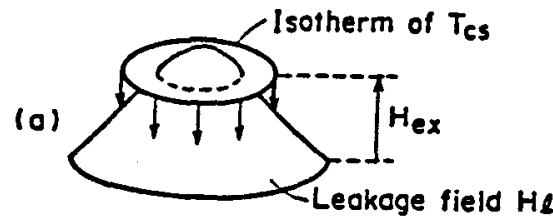

(b)

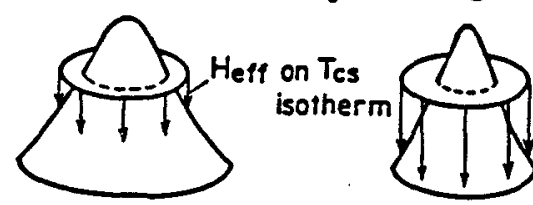

(c)
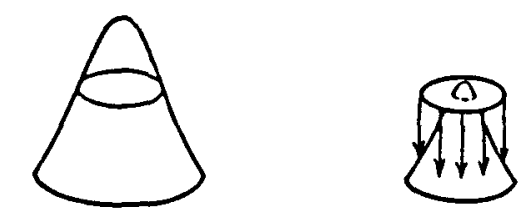

(d)
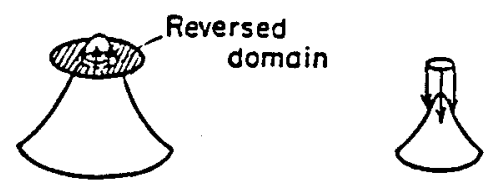

Fig.2 Conceptual view of overwriting process. The drawing like a mountain is the spatial leakage field distribution and the oval is the isotherm of the magnetization reversal temperature of the storage layer. Cooling of the medium progresses as (a), (b), (c), (d).

\section{EXPERIMENTAL}

The film structure is shown in Fig.1. All the layers were deposited by RF magnetron sputtering except the Co layer, which was deposited by DC mode, both under the pressure of 5 mTorr. The sputtering gas was $\mathrm{Ar}+\mathrm{N}_{2}$ for $\mathrm{Si}-\mathrm{N}$ and Ar for the others. The sample was exposed to air after TCL was deposited. A 60 $\mathrm{nm}$ sputter-ctched $\mathrm{Si}-\mathrm{N}$ underlayer and a $5 \mathrm{~nm}$ Pt buffer layer were stacked preliminarily in order to obtain sufficient $\mathrm{Hc}$ for $\mathrm{Co} / \mathrm{Pt}$. The number of $\mathrm{Co} / \mathrm{Pt}$ units, $\mathrm{n}$, was varied from 1 to 7. The magnetic characteristics were measured by a vibrating sample magnetometer using samples on a Si wafer. A recording test was carried out by Nakamichi OMS-2000 using the samples on a pre-grooved $1.2 \mathrm{~mm}$ thick glass disk substrate. The wavelength of the laser was $830 \mathrm{~nm}$, and the linear velocity and the pulse width were $9 \mathrm{~m} / \mathrm{s}$ and $400 \mathrm{~ns}$, respectively.

\section{DESIGN OF Co/Pt MULTILAYERS}

\section{Coercivity and the Magnetization Reversal Temperature}

The film temperature at which Hc becomes zero was lower than the Curic point for the $\mathrm{Co} / \mathrm{Pt}$, as shown later in Fig.5. So, the former temperature was regarded as the magnetization reversal tempcrature $T_{r}$. Assuming magnetization reversal as a magnetic wall motion, this tempcrature was thought to be a better index for Tr than the Curie point.

It has been found that both $\mathrm{Tr}$ and $\mathrm{Hcn}$ were controlled by the Co thickness. The results are shown in Fig.3 for $n=1$ to 5 . $T_{r}$ and $H c n$ decreased with decreasing the thickness irrespective of $\mathrm{n}$. $\mathrm{T}_{\mathrm{r}}$ was found to decrease down to $100^{\circ} \mathrm{C}$, and a $\mathrm{Tr}$ value of about $200^{\circ} \mathrm{C}$ was obtained with sufficient $\mathrm{Hcn}$ for $\mathrm{n}=2$ to 5 . These $T_{r}$ values were lower than those reported so $\mathrm{far}[5]$. $\mathrm{Tr}$ also decreased by making the $\mathrm{Pt}$ layer thicker, but the $\mathrm{Co} / \mathrm{Pt}$ multilayer became thick to obtain sufficient $\mathrm{Hcn}$ in this case. A thick SL is not good for a switching field as will be described later. It is interesting that even a single Co layer sample showed a similar Co thickness dependence as a multilayer.

\section{Switching Field}

A rewritable-mode write/erase test was carried out for the samples without TCL, BL, and IL. Typical results of the dependence of carrier and noise levels on Hex for samples with $n=3$ and $n=7$ are shown in Fig.4. the write power was $6 \mathrm{~mW}$. Signals written under an external field indicated as "W" could be erased completely by the erase power (PE) and Hex 


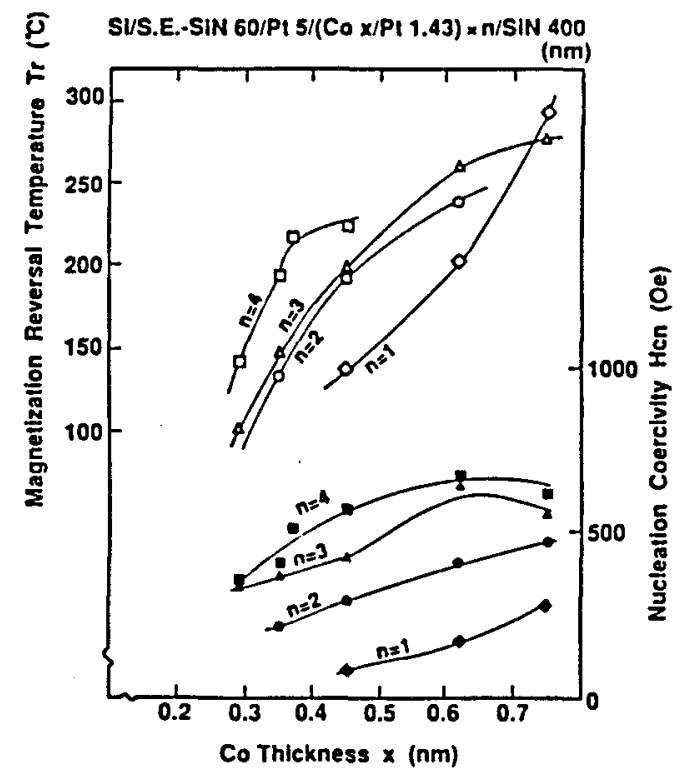

Fig.3 Co thickness dependence of $\operatorname{Tr}$ and Hen for samples with number of $\mathrm{Co} / \mathrm{Pt}$ units $\mathrm{n}=1$ to 5

glass/S.E.-SiN 60/Pt 5/(Co X/Pt 1.43) ×n/SiN $40(\mathrm{~nm})$

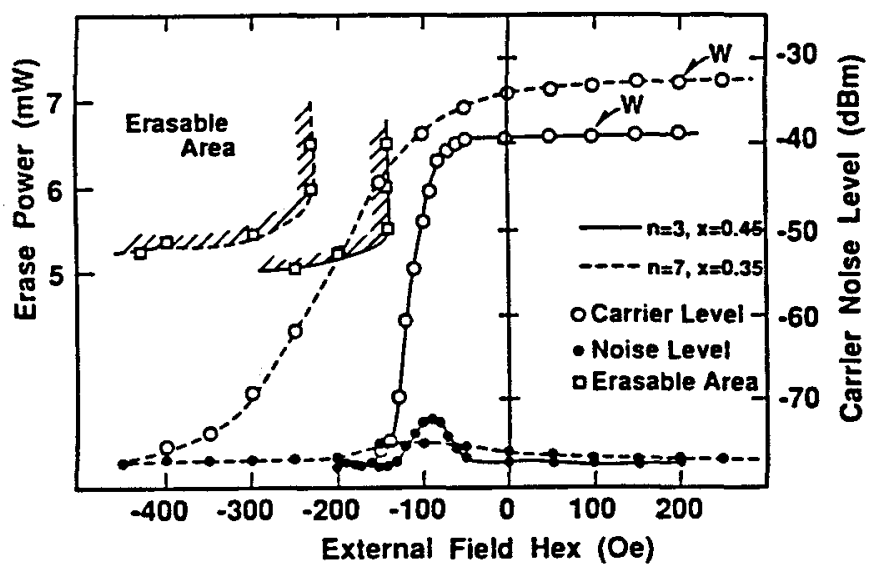

Fig.4 Hex dependence of carrier level and noise level for $\mathrm{n}=3$ and $\mathrm{n}=7$.

within the hatched area. The difference in Hex between the threshold and saturation (named as $\Delta \mathrm{Hsat}$ ) and the switching field $\Delta \mathrm{Hsw}$ for $\mathrm{n}=3$ were found to be smaller than those for $n=7$.

The results of $\Delta \mathrm{Hsat}$ and $\Delta \mathrm{Hsw}$ are summarized in Table 1. It has been found that the thinner the total thickness becomes the smaller $\Delta \mathrm{Hsw}$ becomes. Writing and erasing could be completed by an Hex change of only 80 Oe for the $n=2$ sample. Considering that the demagnetization field is proportional to magnetization and film thickness, the reduction in the demagnetization field in the thinner sample brought a small $\Delta$ Hsat and resulted in a small $\Delta$ Hsw. Samples with $n=2$ to 4 were selected for SL according to the qualification (2).
Tab. 1 Result of recording test

\begin{tabular}{|c|c|c|c|c|}
\hline \multirow[t]{2}{*}{$\mathbf{n}$} & \multicolumn{2}{|c|}{ Thickness (nm) } & \multirow{2}{*}{$\begin{array}{l}\Delta \text { Hsw } \\
\text { (Oe) }\end{array}$} & \multirow{2}{*}{$\begin{array}{l}\Delta \text { Hsat } \\
(\mathrm{Oe})\end{array}$} \\
\hline & Co & $\mathrm{Pt}$ & & \\
\hline$\overline{2}$ & $\overline{0.45}$ & 1.43 & 80 & 35 \\
\hline 3 & 0.45 & 1.43 & 90 & 50 \\
\hline 4 & 0.35 & 1.43 & 90 & 70 \\
\hline 5 & 0.31 & 1.43 & 150 & 130 \\
\hline
\end{tabular}

\section{OVERWRITE EXPERIMENT}

A $200 \mathrm{~nm}$ thick (Gd0.75Tb0.25)0.18(Fe0.97Co0.03)0.82 and a $200 \mathrm{~nm}$ thick (Gd0.5Tb0.5)0.26(Fe0.5 Co0.5)0.74 wereemployed for $\mathrm{BL}$ and $\mathrm{IL}$, respectively. The change in the magnetization $\mathrm{Ms}$ and $\mathrm{Hc}$ against temperaturefor $\mathrm{BL}$ and $\mathrm{Co} / \mathrm{Pt} \mathrm{SL}$ are shown in Fig.5. Ms at ambient temperature and the Curie temperature of $\mathrm{BL}$ were $90 \mathrm{emu} / \mathrm{cc}$ and $220^{\circ} \mathrm{C}$, respectively. $\operatorname{Tr}$ was somewhat lower than the required value according to the qualification (1). It has been confirmed from other experiments that magnetization reversal in $\mathrm{BL}$ is completely suppressed during overwriting by the interfacial wall energy between $B L$ and IL.

The result of a rewritable-mode recording test for the $n=4$ sample is shown in Fig.6. When $\mathrm{Pw}$ was high, the leakage field from $\mathrm{BL}$ became large and the Hex-CNR curve shifted toward the negative Hex direction. From this result, equi-CNR lines on the Hex-Pw plane can be obtained and the degrec of lcakage ficld modulation can be estimated[6]. The degrec of leakage field modulation was found to be about $80 \mathrm{Oe}$ in this case. This value agrees well with the evaluation from numerical calculation[4], 90 Oe. It has been found that $\triangle$ Hsat becomes larger than that of SL alone by stacking $\mathrm{BL}$ and IL, compared with Fig.4. A large $\triangle H$ sat was also observed for the same medium but using a non-magnetic pseudo biasing layer[6]. The change in thermal response of SL is thought to

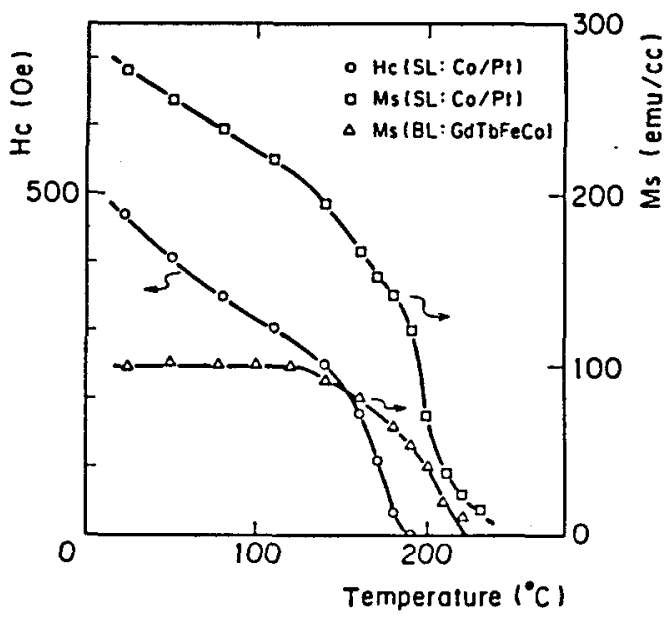

Fig.5 Temperature dependence of magnetization of $\mathrm{BL}$ and of magnetization and coercivity of SL 


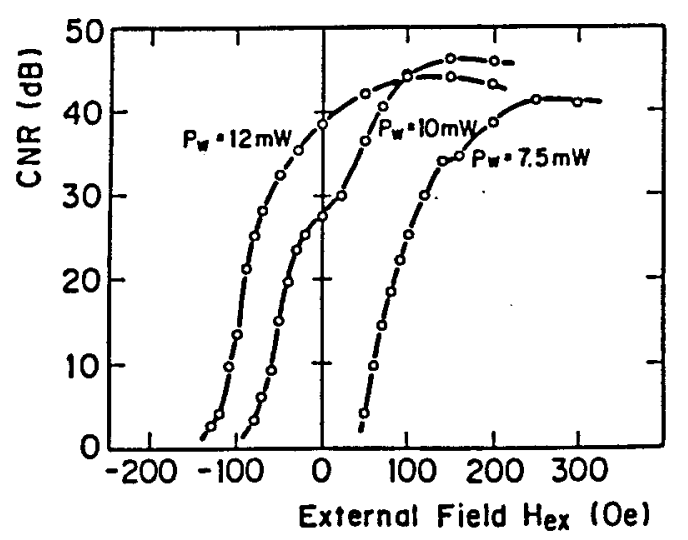

Fig.6 Hex dependence of CNR of $n=4$ sample as a function of writing power

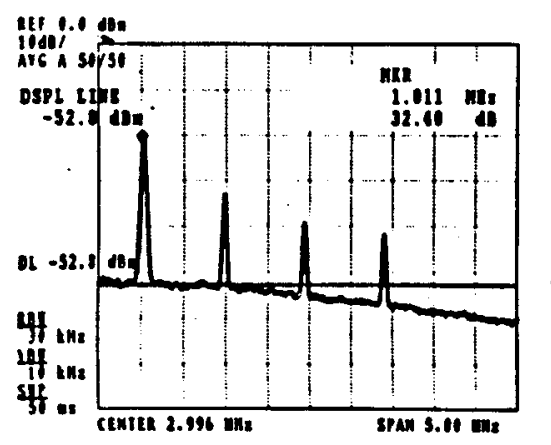

\section{$1.25 \mathrm{MHz}$}

$\rightarrow 1 \mathrm{MHz}$

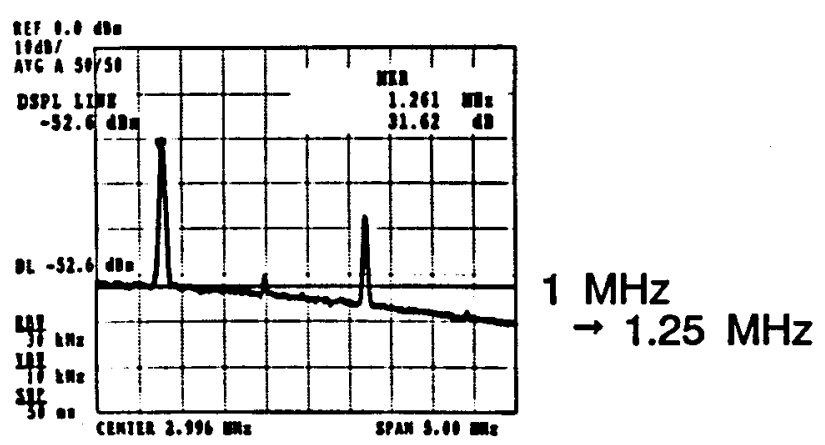

Fig.7 Spectra of overwritten siguals of $n=4$ sample. Peak power was $13 \mathrm{~mW}$, bottom power was $9.4 \mathrm{~mW}$ and Hex was -30 Oe.

make $\triangle$ Hsat larger.

The spectra of overwritten signals for the $\mathrm{n}=4$ sample are shown in Fig.7. The recording conditions are as follows; peak power: $13 \mathrm{~mW}$, bottom power:9.4 $\mathrm{mW}$ and Hex:-30 Oe. These conditions were selected so that the thermal interference between the written marks was reduced in order to confirm the capability of overwrite. Overwrite between $1 \mathrm{MHz}$ and 1.25 $\mathrm{MHz}$ was carried out successfully with CNR 32 $\mathrm{dB}$. OW was also possible for all the other samples with over $30 \mathrm{~dB}$. The OW CNR and the Kerr rotation $\theta \mathrm{k}$ of these samples are listed in Table 2. A large OW CNR was expected for the thinner samples which had small $\Delta \mathrm{Hsw}_{\mathrm{s}}$ values, but the decrease in $\theta \mathrm{k}$, which was due
Tab. 2 Result of overwriting test

\begin{tabular}{|c|c|c|c|}
\hline $\mathbf{n}$ & $\begin{array}{l}\text { OW CNR } \\
\text { (dB) }\end{array}$ & $\begin{array}{cc}\text { Kerr Rotation } \\
\theta_{\boldsymbol{\theta}} & \text { (deg.) }\end{array}$ & $\begin{array}{l}\sqrt{R} \theta_{k} \\
\text { (deg.) }\end{array}$ \\
\hline$\overline{2}$ & 30 & 0.14 & 0.10 \\
\hline 3 & 32 & 0.18 & 0.14 \\
\hline 4 & 32 & 0.24 & 0.17 \\
\hline
\end{tabular}

to the thin Co layer, reduced the CNR of SL and the OW CNR.

In the present study, the OW CNR was lower than the saturated CNR shown in Fig.6, but it is expected to be improved by the following modifications. Leakage field modulation is thought be improved by using a biasing layer with a larger Ms than that in this paper. Such a BL is available as used in a previous study[6]. Tr seems to be somewhat low for overwrite as mentioned above. The leakage field will be used more effectively with a proper adjustment of $\mathrm{T}$.

\section{CONCLUSION}

Laser power modulation overwrite on a $\mathrm{Co} / \mathrm{Pt}$ multilayer was successful by the magneto-statically coupled method. The magnetization reversal temperature and the switching field were modified by changing the Co layer thickness and the number of $\mathrm{Co} / \mathrm{Pt}$ units, respectively, in order to apply $\mathrm{Co} / \mathrm{Pt}$ to OW medium. An overwrite CNR up to $32 \mathrm{~dB}$ was achieved for thin Co/Pt films stacked 2 to 4 times. The OW CNR is expected to increase by some modifications of the medium. The advantages and the capability in the future development of the magneto-statically coupled overwriting method has been demonstrated.

\section{REFERENCE}

[1] K.Ichihara, S.Ashida, A.Kikitsu and Y.Mizusawa : Proc.MORIS'91, J.Magn.Soc. Jpn., 15 Suppl. S1, 1991, pp.315-318

[2] W.B.Zeper, F.J.A.M.Greidanus, P.F.Carcia and C.R.Fincher : J.Appl.Phys., 65, 1989, pp.4971-4975

[3] C.J.Lin and H.V.Do : IEEE Trans.Magn., 26, 1990, pp.1700-1702

[4] A.Kikitsu, S.Ashida, Y.Mizusawa and K. Ichihara : Jpn.J.Appl.Phys., 31, Part 1, No.2B, 1992, pp.414-419

[5] P.F.Carcia, W.B.Zeper, H.W.van Kestern, B. A.J.jacobs and J.H.M.Spurit :Proc. MORIS'91, J.Magn.Soc.Jpn., 15 Suppl. S1, 1991, pp.151-156

[6] K.Ichihara, S.Ashida, A.Kikitsu and Y. Mizusawa : Jpn.J.Appl.Phys., 31, Part 1, No.2B, 1992, pp.420-425 